\title{
Une nouvelle méthode pour l'évaluation globale des géomatériaux
}

B. TARDIEU

Président Directeur Général, Coyne et Bellier 9, allée des Barbanniers 92632 Gennevilliers Cedex

F. COUR

Président, GEODIA

J. HUGONIN Ingénieur Géologue, Coyne et Bellier
Une nouvelle méthode a été développée par Coyne et Bellier et GEODIA, avec l'aide de l'ANVAR, pour les structures de génie civil où l'influence de la fondation rocheuse est grande : tunnels, grands talus d'excavations, barrages, ...

Elle a pour objectif d'aider l'ingénieur, dès le stade préliminaire des études, à comprendre le rocher sur lequel il veut construire, ou dans lequel il veut excaver, ou auquel il s'intéresse pour toute autre raison pratique; l'aider à détecter les éventuelles faiblesses ou anomalies. à caractériser ce site par rapport à d'autres sites connus : enfin dans certains cas, lui fournir des ordres de grandeur de certains paramètres physiques. La méthode s'appuie sur l'analyse fine des signaux acoustiques transmis par le rocher. Elle met en cuvre pour ce faire :

- un équipement léger d'acquisition des enregistrements de terrain,

- un ensemble de procédures normalisées de traitement des données et d'interprétation primaire,

- une importante base de données expérimentales. accumulée depuis plus de trente ans dans toutes sortes de contextes géotechniques.

La méthode est utilisable, sous diverses formes pratiques, chaque fois qu'il faut évaluer la qualité d'un site rocheux. Elle s'applique également à d'autres géomatériaux (béton de masse, béton compacté) pour lesquels la base expérimentale est en cours de constitution.

\section{A new method for a global appraisal of geomaterials}

A new method for a preliminary diagnosis of rock foundation conditions of various large civil engineering works (tunnels, excavations, dams...) has been developed by Coyne et Bellier and Geodia with the assistance of the ANVAR Agency, It is mainly devoted to support the project engineer who has to u appraise $\gg$ the rock foundation at the early beginning of the preliminary study stage. It contributes to the detection of potential weaknesses or abnormal findings, the characterization of the site foundation with regard to that of several reference sites. In addition, it provides values of some physical parameters that characterize the foundation.

The method is based on the detailed analysis of acoustic signals. that are transmitted through the rockmass. It consists of :

- a light field seismic recorder,

- standard operation rules for field data processing and preliminary interpretation of the results,

- a wide experimental data base deriving from thirty years of application on many various geotechnical contexts.

The method can be used as soon as rock mass quality is to be assessed. Similarly, it can be applied for evaluation of other materials, such as : mass concrete and compacted concrete for which the experimental data base is still under development. 


\section{Faire connaissance avec le site d'un futur ouvrage}

L'ingénieur en charge d'un projet nouveau doit très rapidement apprécier les capacités du site sur lequel il entend construire, s'il veut éviter de se fourvoyer dans des solutions qui pourraient apparaître ultérieurement inadéquates. L'image qu'il se fait de ce site est d'abord globale, et s'affine progressivement au fur et à mesure que s'accumulent des indices de différentes natures: morphologie, observation de la roche et des discontinuités diverses en affleurements, en galeries ou en sondages ; puis résultats chiffrés des reconnaissances et essais.

Outre l'observation, le premier « dialogue » avec un massif rocheux se fait à travers le marteau du géologue, puis avec des profils de sismique réfraction: deux approches ( sonores $)$, et cependant très différentes. La sismique fournit presque exclusivement la vitesse de transmission qui est associée de manière imprécise à la déformabilité. Le marteau répond plutôt par un timbre, c'est-à-dire par la forme des ondes en retour du choc; leurs relations avec les qualités du massif sont encore plus floues. Et cependant on sait, par une pratique très ancienne, que ces deux modes de réponse sonore du massif rocheux sont en relation avec ses propriétés mécaniques.

La démarche naturelle de l'ingénieur consiste à comparer, consciemment ou non, l'ensemble des informations acquises avec ce qu'il a vu ou " entendu » ailleurs, sur d'autres sites, ou bien sur d'autres ouvrages en projet ou en exploitation. II se réfère alors à des exemples satisfaisants, où tout va bien, mais aussi et surtout à des cas connus où la géotechnique s'est révèlée source d'ennuis. L'incertitude est bien entendu maximale au démarrage des projets, lorsque les reconnaissances sont partielles voire inexistantes et que les données chiffrées font encore défaut.

Dès l'origine, la (" petite sismique )) (B. Schneider, 1967, 1970, 1990; et al., 1992), dont la nouvelle méthode hérite, a cherché à reproduire cette démarche de rapprochement des observations, en la formalisant et en s'appuyant sur les mesures sonores. Son ambition limitée - est de foumir une évaluation globale et générale mais précoce des qualités d'un site, et non pas d'établir une cartographie de telle ou telle propriété (comme le font d'autres méthodes). L'objectif étant d'orienter rapidement le choix du parti, et d'optimiser les reconnaissances lourdes ultérieures.

Il n'est pas possible d'établir, de manière déterministe, la relation entre la réponse sonore et les propriétés mécaniques du massif parcouru. Seules sont connues des tendances générales entre, par exemple, la vitesse de propagation du son et la déformabilité du massif. En revanche, cette ignorance peut être progressivement comblée, si l'on prend soin de rapprocher systématiquement les mesures sonores effectuées sur toutes sortes de sites, avec les qualités des massifs rocheux appréciées ou mesurées par d'autres moyens.

La démarche, qui est celle adoptée dès le début des années 60 , est beaucoup plus naturaliste, ou heuristique, que déterministe. Elle exige, pour être justifiée, la constitution d'un grand nombre d'expérimentations dans les différents contextes qui couvrent le domaine de pertinence recherché. Ceci explique le relatif degré de confidentialité dans lequel la méthode est restée confinée pendant une trentaine d'années. Ajoutons qu'au début la mise en application s'est trouvée également freinée par les faibles performances de l'appareillage électronique de terrain. Cependant, au fil des années une importante base d'expérience s'est accumulée: à la fin des années 80 , plus de 50 sites avaient fait l'objet de campagnes de "petite sismique», dans le cadre de projets de barrages, tunnels, mines, etc.

\section{2}

\section{Le développement de la méthode}

La richesse de cette base expérimentale méritait d'être mieux exploitée, par une modernisation du matériel de mesure ; par ailleurs, seule une large diffusion de la méthode permettrait d'accroître rapidement la base expérimentale et d'augmenter la valeur de la méthode. Ceci impliquait une solide rationalisation des procédures, à toutes les étapes de la mesure et de l'interprétation

C'est ce que les promoteurs de la méthode ont décidé d'entreprendre. L'opération, qui est maintenant terminée pour l'essentiel, a comporté les étapes suivantes :

- redéfinition du contenu et des procédures de mesures de terrain,

- définition du matériel de terrain, conception et construction de prototypes, expérimentation,

- standardisation des procédures de calcul et d'interprétation primaire,

- contrôle et homogénéisation de la base expérimentale (notamment par réalisation de nouvelles mesures sur des sites connus),

- élaboration des règles d'exploitation de la base de données, à partir d'analyses statistiques,

- développement des logiciels adéquats pour l'analyse du signal, l'interprétation primaire, et le système expert SCARABEX.

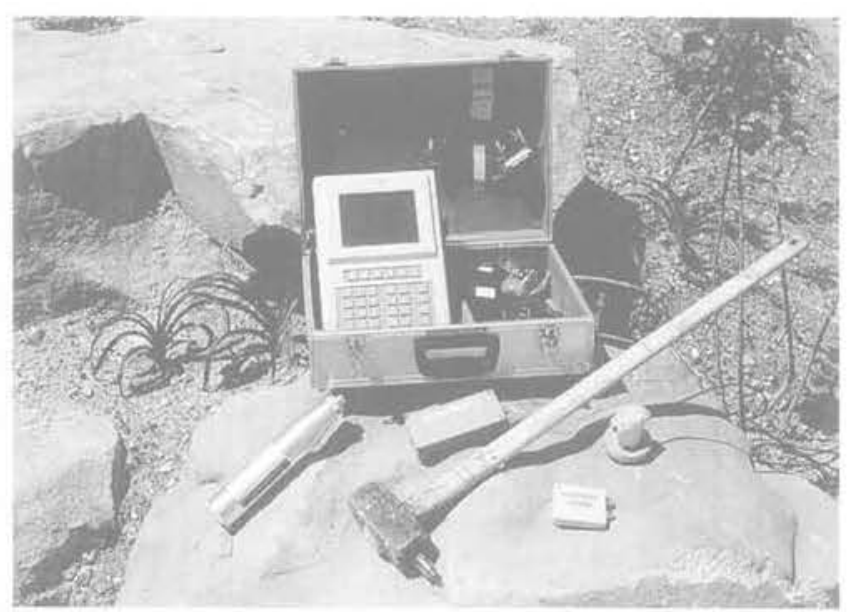

FG.1 Le matériel de terrain. Devant l'enregistreur, on distingue (de gauche à droite) le scléromètre, Yaccéléromètre fixé sur une masse, les conditionneurs de signaux et le géophone. The field measuring equipment.

In front : the recorder; from left to right : sclerometer, accelerometer fixed on sledgehammer, signals conditionners, geophone. 
Le résultat se présente aujourd'hui sous la forme d'un ensemble complet d'appareils, d'accessoires, de notices qui précisent le détail des procédures à appliquer pour toute nouvelle campagne, et de logiciels d'aide à l'interprétation qui peuvent être mis en œuvre sur tout micro-ordinateur au standard du marché. Le tout est assez léger pour être emporté par l'opérateur en bagages à main, et peut être utilisé après une formation rapide (Fig. 1).

\section{3}

\section{Les mesures sur le terrain}

La mise en œuvre sur le terrain doit suivre une méthodologie qui permet une exploitation ultérieure optimale en garantissant l'homogénéité des mesures avec celles des autres campagnes.

Une campagne s'articule autour d'un certain nombre de profils qui sont développés le long d'affleurements rocheux, à l'air libre ou en galeries. La longueur d'un profil est de 30 mètres en moyenne (Fig. 2).

Un minimum de 6 profils est nécessaire pour réduire l'aléa métrologique, dû principalement aux variations géotechniques locales.

Un plus grand nombre de profils permet d'identifier, jusqu'à l'interprétation finale, des groupes de profils réalisés dans des conditions homogènes : compartiments géologiques différents, surface/profondeur d'un massif, etc.

Sur chaque profil, des signaux sismiques sont émis successivement en des points espacés de 3 à 5 mètres, par le choc calibré d'une masse standard (qu'on peut trouver partout). Ils sont reçus en un point fixe à l'extrémité du profil, par un géophone $(30 \mathrm{~Hz})$, puis conditionnés, numérisés et enregistrés avec l'appareillage spécial. La durée du signal enregistré, la bande passante, l'addition éventuelle de coups successifs, sont définis avec précision. Le dispositif permet la visualisation immédiate du signal reçu, afin de pouvoir éliminer les enregistrements aberrants ou perturbés.

A ces mesures purement sismiques, s'ajoute la mesure de la réaction de la roche au scléromètre de Schmidt; les indications de cet appareil représentent la qualité de la matrice, sans tenir compte des discontinuités, puisque cet essai intéresse un tout petit volume.

L'ensemble des manipulations est effectué par un seul opérateur, assisté d'un manœuvre. L'expérience montre que dans des conditions normales d'accessibi-

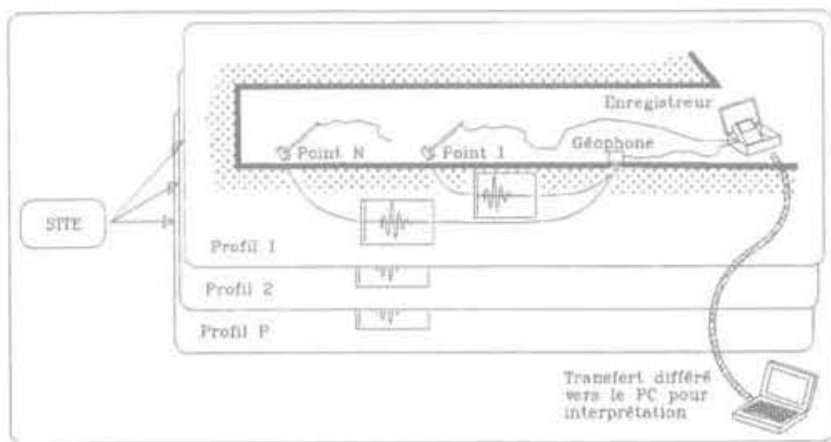

FIG.2

Organisation des mesures de terrain. Field measurements organization. lité il est possible de réaliser en une journée les mesures correspondant à 3 ou 4 profils.

\section{Interprétation des mesures}

Au cours de cette phase, les mesures de terrain sont caractérisées de manière résumée par quelques grandeurs numériques, d'abord à l'échelle du point, puis du profil, puis globalement du site entier (Fig. 3). Ceci est réalisé pratiquement de manière interactive, au moyen d'un ensemble de logiciels dédiés qui fonctionnent sur un micro-ordinateur PC.

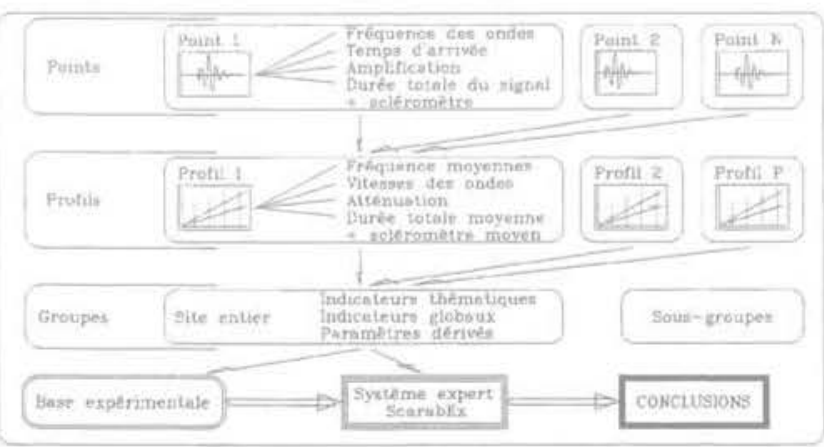

FIG.3 Organisation de l'interprétation. Procedures for results interpretation.

La première opération consiste à transférer les mesures de terrain (sous forme de fichiers de données) depuis l'équipement de terrain vers le micro-ordinateur. L'exploitation est ensuite réalisée en 3 étapes :

\section{1}

\section{Interprétation des signaux élémentaires}

Le signal, enregistré et numérisé pour chaque point, comporte deux ondes bien identifiées (Fig. 4)

- la première, souvent faible et fugace, correspond probablement à la transmission d'une onde de compression,

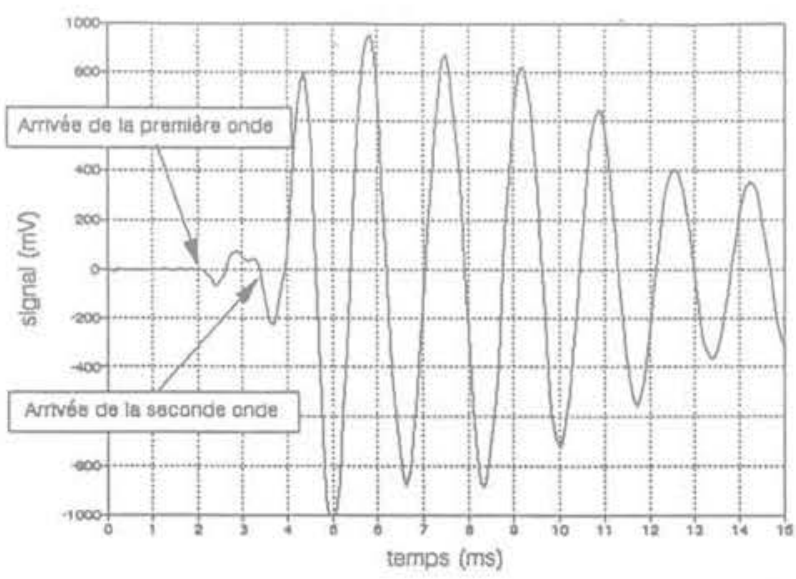

FiG.4 Analyse du signal transmis par le massif. Plotting of the seismic signal that is conveyed through the rockmass. 
- la seconde, plus tardive mais plus forte et plus persistante, domine la fin du signal et caractérise probablement l'onde de cisaillement.

On identifie parfois d'autres composantes, dont la connaissance est pour l'instant insuffisante pour qu'elles puissent être incorporées au processus d'interprétation.

Le signal est décrit de manière résumée par la fréquence, le délai d'arrivée et l'amplification de la première et de la deuxième onde, ainsi que par la durée totale du signal. Le processus d'extraction est effectué sous contrôle permanent de l'opérateur à l'écran, au moyen d'un logiciel spécial.

Par ailleurs, à chaque signal reste associée la valeur sclérométrique au point d'émission correspondant.

\section{2}

\section{Globalisation sur un profil complet}

En rapprochant entre eux les résultats obtenus en chaque point d'un même profil, celui-ci est caractérisé à son tour par plusieurs nombres :

- vitesse de la première et de la deuxième onde, module dynamique Ed et coefficient de Poisson correspondants,

- amplification (gain, G15) et fréquences de la première et de la deuxième ondes (Fp, Fs ; valeurs ajustées à $15 \mathrm{~m}$ ),

- durée totale du signal (valeur ajustée à 15 m),

- indice scléromètrique $\mathrm{S}$ (valeur moyenne).

Les variabilités sont également quantifiées (mais non exploitées).

Par commodité, les paramètres principaux sont normés de telle sorte que la valeur 1 corresponde à une roche moyenne, assez proche du béton de masse; ils fournissent les indicateurs thématiques, ainsi nommés car ils sont porteurs d'une signification physique:

(S) Valeur scléromètre (Unité Schmidt)

$\mathrm{Ks}=\frac{40}{40} \begin{aligned} & \text { caractérise la matrice } \\ & \text { rocheuse }\end{aligned}$

(Ed) Module dynamique (Mpa)
$\mathrm{Ke}=\longrightarrow$ réponse élastique vraie $44000 \quad$ (dynamique)

(Fs) Fréquence fréquence deuxième onde, $2^{\circ}$ arrivée $(\mathrm{Hz})$ représentative du cisaillement, $\mathrm{Kf}=\longrightarrow$ de la plasticité, des déforma750 tions lentes (module statique)

45 gain à $15 \mathrm{~m}$, représente la perte $\mathrm{Ka}=\longrightarrow$ d'énergie dans les disconti(G15s) Amplitude nuités du massif, la fissuration standard $(\mathrm{dB})$ du rocher

On détermine ensuite les indicateurs globaux pour le profil :

- le paramètre Scarabée PS : c'est la moyenne des 4 indicateurs thématiques précédents ; d'une façon générale sa valeur est d'autant plus élevée que la qualité du massif autour du profil est bonne. Le domaine usuel se situe entre 0,5 et 1,5 ;

- le coefficient de variation CvPS des 4 indicateurs thématiques ; c'est un indicateur d'anomalie du massif, tra- duisant que les caractérisations ne sont pas homogènes; les valeurs usuelles se rangent entre 0,05 et 0,50 .

Ces calculs sont effectués automatiquement.

\section{3}

\section{Globalisation au site entier}

La moyenne et la variabilité des indicateurs thématiques sont calculées pour l'ensemble du site, ainsi que pour les indicateurs globaux, dont la stabilité est vérifiée.

Si des variations notables apparaissent, et si le nombre de profils réalisés est suffisant, il est possible d'évaluer les indicateurs pour des groupes de profils, le regroupement étant effectué a priori à partir de données objectives autres du site (position des profils dans l'espace, dans des compartiments géologiques différents), ou bien a posteriori sur des bases purement statistiques en cherchant à minimiser les variations au sein de chaque groupe.

Ces calculs sont effectués automatiquement.

\section{5}

\section{Exploitation de la base expérimentale}

La signification des indicateurs résulte des valeurs qu'ils prennent sur les différents sites ayant fait l'objet d'une expérimentation dans le passé.

La base de mesures expérimentales couvre actuellement plus de 60 sites, 3000 points de mesure, 11000 mètres répartis sur 560 profils.

L'exploitation de la base est facilitée par l'emploi du logiciel ScarabEx, système expert dédié dans lequel ont été introduites les règles issues de l'expérience, dont les conclusions sont gouvernées par les valeurs des indicateurs. Ce système couvre actuellement cinq contextes d'application : les fondations de barrages poids et voùtes, les ouvrages souterrains, les qualités générales d'un massif rocheux, et enfin le contexte " massif en béton », introduit récemment.

Outre cette première approche, il est aussi possible de rechercher plus directement les similitudes avec l'un ou l’autre des sites déjà expérimentés.

\section{6}

\section{Corrélations particulières}

L'application répétée à des contextes particuliers a permis de mettre au point des corrélations entre certains indicateurs et des propriétés particulières des massifs rocheux. Les évaluations les plus utilisées en pratique sont :

- le module statique réversible global du massif, tel que déterminé au moyen de la plaque de charge (Fig. 5),

- le soutènement nécessaire lors de l'excavation de tunnels jusqu'à $6 \mathrm{~m}$ de diamètre.

D'autres corrélations, moins nettes à l'heure actuelle, pourraient donner lieu dans l'avenir à une exploitation enrichie des résultats de la méthode. 


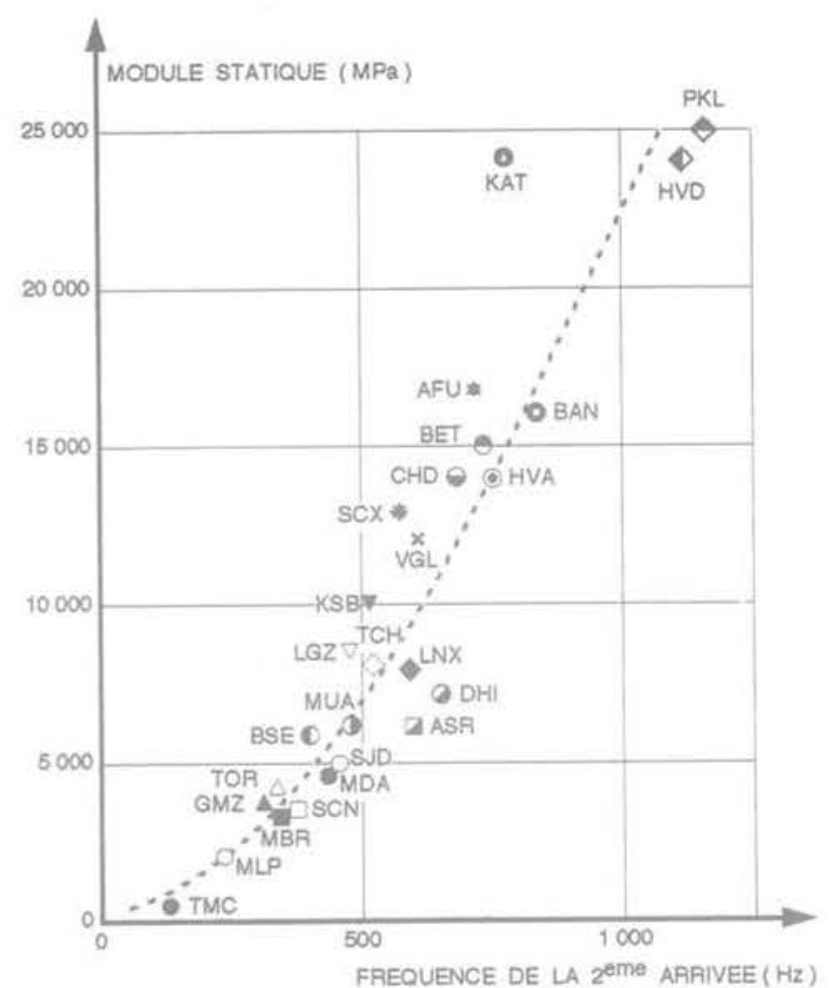

IG.5 Détermination approchée du module statique.

Experimental correlation between elastic moduli and frequencies.

\section{Conclusion}

La méthode proposée fournit un moyen rapide d'appréciation globale d'un massif rocheux. Elle n'a pas l'ambition de remplacer une véritable campagne de reconnaissance géotechnique, mais elle la précède utilement, ne serait-ce que pour en préciser l'orientation.

La mise en œuvre peut intervenir très tôt dans le déroulement du projet, même lorsque l'accessibilité du site aux moyens lourds n'est pas assurée. L'usage des explosifs n'etant pas requis, aucune autorisation administrative n'est nécessaire. Cette méthode permet à l'équipe de projet de détecter sans délai certains pièges, et lui évite ainsi de s'orienter vers un choix de parti inadapté.

Les limites de la méthode découlent de sa résolution modeste (il n'est pas question de faire une cartographie précise des propriétés du site) et aussi de celles du domaine expérimental couvert dans le passé : l'une des conclusions possibles du logiciel expert est « sans précédent connu $n$; mais ce simple message constitue en lui-même un avertissement précieux.

\section{Bibliographie}

Schneider B. - Contribution à l'êtude des massifs de fondation de barrages. Faculté des Sciences de Grenoble. Mémoire $n^{\circ}$ 7, 1967

Schneider B. - Reconnaissance des massifs rocheux par la méthode appelée petite sismique, Bulletin de l'Association Internationale de Géologie de IIngénieur. $n^{\circ} 20,1970$
Bienawski Z.T. - The "Petite Sismique" technique - a review of current developments, procs. 2nd Conference on acoustic emission / microseismic activities in geologic structures and materials, Pennsylvania State University, USA, 1978.

Carrère A., Rivet J., Schneider B. - La Petite Sismicue, Géoloques, n 92, 1992.
Schneider B. - Détermination du module statique et autres caractéristiques géotechniques des fondations rocheuses de barrages par la petite sismique, Mem. Soc. Géol. France, N.S. n 157 , ard, 1990

Bouvard A. Hugonin J., Schneider B, Scarabée: méthode de reconnaissance des massifs rocheux, application aux ouvrages souterrains, Revue de l'AFTES, mars 1994 